Research Paper

\title{
Elevated baseline serum lactate dehydrogenase indicates a poor prognosis in primary duodenum adenocarcinoma patients
}

\author{
Zhan-hong Chen ${ }^{1,2 *}$, Miao-zhen Qiu1*, Xiang-yuan $\mathrm{Wu}^{2 *}$, Qi-nian $\mathrm{Wu}^{1}$, Jia-huan $\mathrm{Lu}^{1}$, Zhao-lei Zeng1, Yun
} Wang1, Xiao-li Wei ${ }^{1}$, Feng Wang ${ }^{1 凶}$, Rui-hua $\mathrm{Xu}^{1 凶}$

1. Department of Medical Oncology of Sun Yat-sen University Cancer Center, State Key Laboratory of Oncology in South China, Collaborative Innovation Center for Cancer Medicine,651 Dongfengdong Road, Guangzhou,510060, China

2. Department of Medical Oncology and Guangdong Key Laboratory of Liver Disease, the Third Affiliated Hospital of Sun Yat-sen University, 600 Tianhe Road, Guangzhou,510630, People's Republic of China

* These authors contributed equally to this work

$\square$ Corresponding authors: Feng Wang: Address: Department of Medical Oncology of Sun Yat-sen University Cancer Center, State Key Laboratory of Oncology in South China, Collaborative Innovation Center for Cancer Medicine, 651 Dongfengdong Road, Guangzhou 510060, China; Tel: 86-20-87342297; Fax: +86-20-87343392; Email: wangfeng@sysucc.org.cn and Rui-hua Xu: Address: Department of Medical Oncology of Sun Yat-sen University Cancer Center, State Key Laboratory of Oncology in South China, Collaborative Innovation Center for Cancer Medicine, 651 Dongfengdong Road, Guangzhou 510060, China; Tel: 86-20-87343795; Fax: +86-20-87343392; Email: xurh@sysucc.org.cn

(c) Ivyspring International Publisher. This is an open access article distributed under the terms of the Creative Commons Attribution (CC BY-NC) license (https://creativecommons.org/licenses/by-nc/4.0/). See http://ivyspring.com/terms for full terms and conditions.

Received: 2017.08.09; Accepted: 2017.12.02; Published: 2018.01.01

\begin{abstract}
Purpose: Tumour cells produce energy through glycolysis and lactate dehydrogenase (LDH) is a key part of glycolysis. Elevation of serum LDH may indicate poor prognosis in primary duodenum adenocarcinoma. We aim to explore the prognostic significance of LDH in this disease.

Methods and materials: Two hundred forty-four patients diagnosed with primary duodenum adenocarcinoma who were treated at the Sun Yat-sen Cancer Center from February 1996 to January 2016 were retrospectively analysed. We collected routine clinical data, including baseline LDH. Patients were classified into a normal LDH group $(\leq 245 \mathrm{U} / \mathrm{L})$ and higher $\mathrm{LDH}$ group $(>245 \mathrm{U} / \mathrm{L})$. Correlations of the LDH level and other clinicopathological characteristics were explored using the Chi-square test. Prognostic factors for overall survival were identified using univariate and multivariate analyses.

Results: Two hundred seven patients (84.9\%) had normal LDH levels, while 37 patients (15.1\%) had abnormally high LDH levels. Higher LDH levels were significantly associated with more distant metastasis, node metastasis, poor differentiation and TNM stage III-IV $(P<0.05)$. Consistently, patients with node metastasis, poor differentiation and TNM stageIII-IV had a significantly higher median LDH level $(\mathrm{P}<0.05)$. The median survival of patients in the higher LDH group was significantly shorter than that of the patients in the normal LDH group ( $16.3 \mathrm{~m}$ vs. $42.5 \mathrm{~m}, P=0.02)$. Using multivariate analysis, $\mathrm{LDH}$, age and radical surgery were independent prognostic factors associated with overall survival $(O S)(H R=1.571$, $P=0.036$ for $L D H ; H R=1.514, P=0.013$ for age; $H R=0.248, P<0.0001$ for radical surgery, respectively).

Conclusions: For the first time, our research suggests that baseline serum LDH is an independent prognostic factor in primary duodenum adenocarcinoma patients and elevated baseline serum LDH indicates a poor prognosis.
\end{abstract}

Key words: lactate dehydrogenase; primary duodenum adenocarcinoma; prognosis; serum biomarker

\section{Background}

Primary duodenum adenocarcinoma is a rare tumour with a very low estimated incidence rate $(<0.5 / 100,000)[1-2]$. It makes up approximately half of small bowel cancers and approximately $1 \%$ of all gastrointestinal cancers [3]. Due to a lack of specific duodenal adenocarcinoma symptoms, a portion of 
patients are diagnosed at the metastatic stage [4]. The median overall survival(OS) of metastatic duodenum adenocarcinoma, ranging from 2 to 8 months, is much worse than advanced colorectal cancer [4]. Various studies have attempted to evaluate different prognostic factors for primary duodenum adenocarcinoma, and some studies have identified the $\mathrm{T}$ stage, nodal metastasis, pathologic grade, lymphovascular invasion, age, weight loss and perineural invasion as prognostic factors [5-12]. Due to the rare incidence, the number of patients in previous studies was relatively small, with a range from 17 to 178 patients [5-12]. However, none of these studies identified any prognostic serum biomarker.

The metabolism of tumour cells has its own characteristics. To coincide with the rapid development of the tumour, tumour cells produce energy through glycolysis. We call this the Warburg effect. This alternation of cancer cell metabolism is believed to be a main change in neoplastic transformation [13]. The hypoxic microenvironment is another feature of malignant tumours. In conditions of hypoxia, lactate dehydrogenase (LDH) can convert pyruvate to lactate to support tumour cells. Hypoxia inducible factor 1a (HIF-1a) can transcriptionally upregulate $\mathrm{LDH}$ in tumour cells to ensure anaerobic glycolysis and produce enough lactate [14]. High levels of lactate can in turn promote higher expression of HIF-1a. Thus, HIF-1a and LDH interact and strengthen each other [14]. Furthermore, higher LDH is significantly correlated with increased vascular density and decreased infiltration of lymphocytes within the tumours. Therefore, the increased LDH level reflects these alterations and suggests more severe tumour burden, tumour angiogenesis and tumour progression conditions, all of which lead to the poor prognosis of malignant tumours [15].

Elevated serum LDH has been confirmed as a poor prognostic factor in many types of malignancies, such as renal cell cancer, nasopharyngeal carcinoma, pancreatic cancer, lymphoma, breast cancer, prostate cancer, colorectal cancer, lung cancer and oesophageal squamous cell carcinoma [16-24]. Some researchers even incorporated LDH into the prognostic scores [19, 24]. The serum LDH level is routinely tested in daily clinical practice, which is economical and convenient to perform. It is worthwhile for us to explore the prognostic significance of the serum LDH level in patients with primary duodenum adenocarcinoma.

For the first time, this research investigates the prognostic significance of serum LDH level in a large cohort of 244 patients with primary duodenum adenocarcinoma. The relationship between $\mathrm{LDH}$ and other clinical factors was also explored in the current study.

\section{Methods}

\section{Patient selection and evaluation}

We studied electronic medical records of primary duodenum adenocarcinoma patients hospitalized in Sun Yat-sen Cancer Center from February 1996 to January 2016. Written informed consent was provided by all patients included in our study. The ethics committees of Sun Yat-sen Cancer Center approved our study. We conducted this study and observed the Helsinki declaration.

We included patients who were diagnosed as having primary duodenum adenocarcinoma by pathology. The exclusion criteria is as follows: (1) pathologically diagnosed with other types of tumours, such as gastrointestinal stromal tumour, neuroendocrine carcinoma and lymphoma; (2) patients with tumours of the distal common bile duct, pancreas or the ampulla of Vater; and (3) patients with incomplete clinicopathological factor information, with synchronous malignant tumours or patients who were lost to follow-up within three months. In total, 244 patients were enrolled in our study.

\section{Data collection}

At first diagnosis, we collected clinical data, including gender, age, abdominal pain, smoking history, family history of cancer, white blood cells (WBC), neutrophil, lymphocyte, monocyte, haemoglobin, platelet, albumin, LDH, TNM stage, pathological differentiation, endoscopy examination and imaging results. TNM stages were classified according to the 7th edition of the TNM staging system by the American Joint Committee on Cancer standards(AJCC). We measured the serum LDH after 2 months of therapy. We also included other prognostic factors, such as the platelet-lymphocyte ratio (PLR), lymphocyte-monocyte ratio (LMR), neutrophil-lymphocyte ratio (NLR), and prognostic nutritional index (PNI, PNI $=5 \times$ lymphocyte count $\left(10^{9} / \mathrm{L}\right)+10 \times$ albumin $\left.(\mathrm{g} / \mathrm{dL})\right)$. We regularly followed up patients by phone or using follow-up in the outpatient clinic. The last follow-up date was June $20^{\text {th }}, 2017$. The OS was the primary outcome and defined as the period from diagnosis to the date of death or to the date of the last follow-up.

\section{Statistical analysis}

We performed statistical analysis using SPSS (version 22.0; IBM Corp., Armonk, NY, USA), GraphPad Prism 6.0 (GraphPad Software Inc., La Jolla, CA, USA) and MedCalc software (version 11.4.20; MedCalc Software bvba, Acacialaan, Belgium). We considered a two-tailed $P<0.05$ as 
statistical significance. Cut-off points for the LDH, WBC, haemoglobin and platelet levels were determined using the upper limit of the normal levels, while cut-off values of NLR, PLR, PNI, and LMR were established using the receiver operating characteristic (ROC) curve analysis. The optimal cut-off value had the maximum Youden index (sensitivity + specificity - 1). We compared differences in the categorical factors between the groups using the Chi-square test and Fisher's exact test. Median values between the different groups were compared using the Mann-Whitney test. In the univariate analyses, we estimated the survival difference of the distinct variables using the Kaplan-Meier method (log-rank test) and identified significant factors. We subsequently brought these factors into the Cox proportional hazards regression model in multivariate analysis to distinguish the independent prognostic factors.

\section{Results}

\section{Patient characteristics}

A total of 244 patients with primary duodenum adenocarcinoma were included in our study. Baseline demographics are shown in Table 1. The median age was 57 years old with an interquartile range (IQR) of 49-63 years old. One hundred fifty-four (63.1\%)patients were male and 90 (36.9\%)patients were female. Ninety-five $(38.9 \%)$ patients presented with abdominal pain at the first diagnosis. Fifty-two $(21.3 \%)$ patients were diagnosed with a metastatic tumour and $192(78.7 \%)$ patients had a non-metastatic tumour. Thirty-seven (15.2\%), 86 (35.2\%) and 69 $(28.3 \%)$ patients were diagnosed at stages I, II and III, respectively. One hundred sixty-four $(67.2 \%)$ patients received radical surgery while $80(32.8 \%)$ patients received palliative therapies, such as chemotherapy and radiotherapy. For all included patients, the 1-year, 2-year, 3-year and 5-year survival rates were $75.0 \%, 57.8 \%, 42.6 \%$ and $27.5 \%$, respectively. For patients who received radical surgery, the 1-year, and 2-year, 3-year and 5-year survival rates were 90.2\%, $74.4 \%, 57.3 \%$ and $35.4 \%$, respectively. Eventually, 207 $(84.9 \%)$ patients were classified into the normal LDH $(\leq 245 \mathrm{U} / \mathrm{L})$ group, while $37(15.1 \%)$ patients were in the higher $\mathrm{LDH}(>245 \mathrm{U} / \mathrm{L})$ group.

The median baseline serum LDH levels were significantly higher in patients with node metastases than those without (188.5U/L vs. $171.5 \mathrm{U} / \mathrm{L}, \mathrm{P}=0.01)$. The same can be said for those with stage III-IV compared to those with stage I-II (190U/L vs. $171 \mathrm{U} / \mathrm{L}, \mathrm{P}=0.009)$, as well as those with poor differentiation compared to those with well \& moderate differentiation (190U/L vs. $171 \mathrm{U} / \mathrm{L}$,
$\mathrm{P}=0.018)$ (Figure 1).

Table 1. Baseline demographics of 244 patients with primary duodenum adenocarcinoma.

\begin{tabular}{|c|c|}
\hline Characteristic & No. of cases $(\%$ \\
\hline Age, years (median/IQR) & $57(49-63)$ \\
\hline \multicolumn{2}{|l|}{ Gender } \\
\hline Male & $154(63.1)$ \\
\hline Female & $90(36.9)$ \\
\hline \multicolumn{2}{|l|}{ Abdominal pain } \\
\hline Yes & $95(38.9)$ \\
\hline No & $149(61.1)$ \\
\hline \multicolumn{2}{|l|}{ Smoking history } \\
\hline Yes & $56(23.0)$ \\
\hline No & $188(77.0)$ \\
\hline \multicolumn{2}{|l|}{ Family history of cancer } \\
\hline Yes & $33(13.5)$ \\
\hline No & $211(86.5)$ \\
\hline \multicolumn{2}{|l|}{$\begin{array}{l}\text { Laboratory parameters } \\
\text { (median/ IQR) }\end{array}$} \\
\hline WBC $(* 10 \% / L)$ & $7.4(5.6-10.4)$ \\
\hline Neutrophil (*109/L) & $5.1(3.3-7.5)$ \\
\hline Lymphocyte $\left({ }^{*} 10^{9} / \mathrm{L}\right)$ & $1.4(1.1-1.8)$ \\
\hline Monocyte $\left({ }^{*} 10^{9} / \mathrm{L}\right)$ & $0.5(0.4-0.8)$ \\
\hline Haemoglobin $(\mathrm{g} / \mathrm{L})$ & $108(95-123)$ \\
\hline Platelet $\left({ }^{*} 10 \% / \mathrm{L}\right)$ & $259(186-340)$ \\
\hline Albumin $(g / L)$ & $38(34.5-41.3)$ \\
\hline LDH (U/L) & $176(153-216)$ \\
\hline \multicolumn{2}{|l|}{ T category (AJCC 7th) } \\
\hline T1 & $10(4.1)$ \\
\hline T2 & $61(25.0)$ \\
\hline T3 & $54(22.1)$ \\
\hline T4 & $119(48.8)$ \\
\hline \multicolumn{2}{|l|}{$\mathrm{N}$ category (AJCC 7th) } \\
\hline No & $128(52.5)$ \\
\hline N1 & $92(37.7)$ \\
\hline N2 & $24(9.8)$ \\
\hline \multicolumn{2}{|l|}{ M category (AJCC 7th) } \\
\hline Mo & $192(78.7)$ \\
\hline M1 & $52(21.3)$ \\
\hline \multicolumn{2}{|l|}{ TNM (AJCC 7th) } \\
\hline I & $37(15.2)$ \\
\hline II & $86(35.2)$ \\
\hline III & $69(28.3)$ \\
\hline IV & $52(21.3)$ \\
\hline \multicolumn{2}{|l|}{ Radical surgery } \\
\hline Yes & $164(67.2)$ \\
\hline No & $80(32.8)$ \\
\hline 1-year survival rate & $183(75.0)$ \\
\hline 2-year survival rate & $141(57.8)$ \\
\hline 3-year survival rate & $104(42.6)$ \\
\hline 5-year survival rate & $67(27.5)$ \\
\hline
\end{tabular}

\section{The association between LDH and clinicopathological factors}

We classified all patients into a series of subgroups according to the cut-off values of the different clinicopathological factors and analysed the potential correlations between these factors and the serum LDH level. We found that higher $\mathrm{LDH}$ level(>245U/L) was significantly associated with more distant metastasis, more node metastasis, poorer 
differentiation, more advanced TNM stage(III-IV) and higher neutrophil to lymphocyte ratio (NLR) $(\mathrm{P}<0.05)$. There was a statistical trend between the higher LDH level and older age $(>57$ years old $)(P=0.051)$. We did not find other significant associations between the LDH and other clinicopathological factors listed in Table 2 so far.

\section{Survival analysis}

The median survival of all the patients was 31.3 months. At the end of our study, a total of 148 patients died of primary duodenum adenocarcinoma and 96 patients had survived. Median survival of the patients with a normal LDH level( $\leq 245 \mathrm{U} / \mathrm{L})$ was significantly longer than that of patients with a higher $\mathrm{LDH}$ level(>245U/L) (42.5 m vs. $16.3 \mathrm{~m}, \mathrm{P}=0.02)$. The 1-year, 2-year, 3-year and 5-year survival rates were $77.8 \%, 60.4 \%, 44.9 \%$ and $28.5 \%$, respectively, for patients in the normal LDH group, while the rates were $59.5 \%, 43.2 \%, 29.7 \%$ and $21.6 \%$ for those in the higher LDH group, respectively. As shown in Table 3 and Figure 2, various factors, including age, node metastasis, distant metastasis, radical surgery, TNM stage, degree of differentiation, PNI and LDH were all significantly associated with $\mathrm{OS}(P<0.05)$. However, factors such as gender, $\mathrm{T}$ category (T1-2 vs. T3-4), NLR, PLR and LMR were not associated with survival in the current study $(P>0.05)$. In the multivariate analysis, we brought all the significant variables determined by the univariate analyses into the Cox proportional hazards model. As the results showed, $\mathrm{LDH}$, age and radical surgery were independent prognostic factors associated with the OS $(\mathrm{HR}=1.571$, $P=0.036$ for $\mathrm{LDH}$; HR=1.514, $P=0.013$ for age; $\mathrm{HR}=0.248, P<0.0001$ for radical surgery, respectively).

Based on the serum LDH values before and after 2 months of radical or palliative treatment, we divided these patients into 4 groups: the low-low group $(n=180)$, low-high group $(n=27)$, high-low group $(n=17)$ and high-high group $(n=20)$ (Table 4). The high-high group showed a similar OS with that of the low-high and high-low group (14.5 $\mathrm{m}$ vs $15.4 \mathrm{~m}$ vs $39.3 \mathrm{~m}, P>0.05)$, while the high-low group showed a similar OS with that of the low-low group $(39.3 \mathrm{~m}$ vs $52.1 \mathrm{~m}, P=0.592)$. Patients with elevated LDH before or after 2 months of treatment (High-high/high-low/ low-high group) showed a significantly shorter OS than that of the low-low group (15.9 m vs $52.1 \mathrm{~m}$, $P=0.0003$ ) (Table 4 and Figure 3).

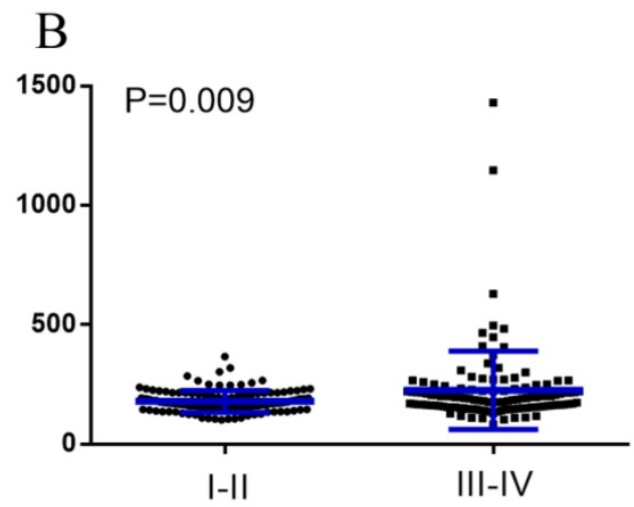

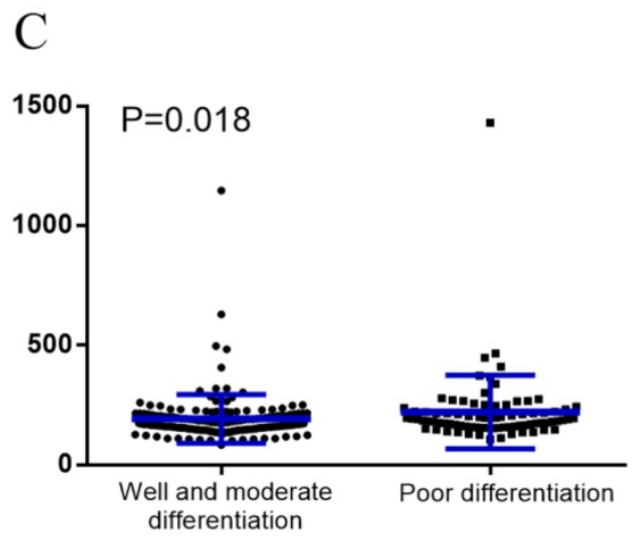

Figure 1. Baseline serum LDH levels were compared using the Mann-Whitney test in different subgroups. (A), Without node metastasis vs. with node metastasis; (B), TNM stage I - II vs. TNM stage III-IV; (C), Well and moderate differentiation vs. poor differentiation. 
Table 2. Correlation analysis for clinicopathologic variables in the normal LDH group and higher LDH group among the 244 primary duodenum adenocarcinoma patients.

\begin{tabular}{|c|c|c|c|}
\hline Variable & $\begin{array}{l}\text { Normal LDH } \\
\leq 245 \mathrm{U} / \mathrm{L} \\
\text { n }(\%)\end{array}$ & $\begin{array}{l}\text { High LDH } \\
>245 \text { U/L } \\
\text { n }(\%)\end{array}$ & $P$ value \\
\hline Total & $207(84.9)$ & $37(15.1)$ & \\
\hline Age, years & & & 0.051 \\
\hline$>57$ & $92(44.4)$ & $23(62.2)$ & \\
\hline$\leq 57$ & $115(55.6)$ & $14(37.8)$ & \\
\hline Gender & & & 0.584 \\
\hline male & $129(62.3)$ & $25(67.6)$ & \\
\hline female & $78(37.7)$ & $12(32.4)$ & \\
\hline WBC & & & 0.315 \\
\hline$>10 * 109 / \mathrm{L}$ & $53(25.6)$ & $13(35.1)$ & \\
\hline$\leq 10 * 109 / \mathrm{L}$ & $154(74.4)$ & $24(64.9)$ & \\
\hline Haemoglobin & & & 0.695 \\
\hline$>120 \mathrm{~g} / \mathrm{L}$ & $58(28.0)$ & $9(24.3)$ & \\
\hline$\leq 120 \mathrm{~g} / \mathrm{L}$ & $149(72.0)$ & $28(75.7)$ & \\
\hline Platelet & & & 1 \\
\hline$>300 * 10 \% / \mathrm{L}$ & $73(35.3)$ & $13(35.1)$ & \\
\hline$\leq 300 * 109 / \mathrm{L}$ & $134(64.7)$ & $24(64.9)$ & \\
\hline NLR & & & 0.022 \\
\hline$>2.56$ & $131(63.3)$ & $31(83.8)$ & \\
\hline$\leq 2.56$ & $76(36.7)$ & $6(16.2)$ & \\
\hline PLR & & & 0.673 \\
\hline$>267.5$ & $47(22.7)$ & $10(27)$ & \\
\hline$\leq 267.5$ & $160(77.3)$ & $27(73)$ & \\
\hline PNI & & & 0.216 \\
\hline$>45.7$ & $102(49.3)$ & $14(37.8)$ & \\
\hline$\leq 45.7$ & $105(50.7)$ & $23(62.2)$ & \\
\hline LMR & & & 0.521 \\
\hline$>4.7$ & $44(21.3)$ & $6(16.2)$ & \\
\hline$\leq 4.7$ & $163(78.7)$ & $31(83.8)$ & \\
\hline $\mathrm{T}$ category & & & 0.239 \\
\hline $1-2$ & $57(27.5)$ & $14(37.8)$ & \\
\hline 3-4 & $150(72.5)$ & $23(62.2)$ & \\
\hline $\mathrm{N}$ category & & & 0.012 \\
\hline 0 & $116(56.0)$ & $12(32.4)$ & \\
\hline $1-2$ & $91(44.0)$ & $25(67.6)$ & \\
\hline M category (M0/M1) & & & 0.031 \\
\hline 0 & $168(81.2)$ & $24(64.9)$ & \\
\hline 1 & $39(18.8)$ & $13(35.1)$ & \\
\hline Degree of differentiation & & & 0.037 \\
\hline Well and moderately differentiated & $144(69.6)$ & $19(51.4)$ & \\
\hline Poorly differentiated & $63(30.4)$ & $18(48.6)$ & \\
\hline TNM stage & & & 0.007 \\
\hline I-II & $112(54.1)$ & $11(29.7)$ & \\
\hline III-IV & $95(45.9)$ & $26(70.3)$ & \\
\hline
\end{tabular}

LDH: lactate dehydrogenase; TNM: Tumour-node-metastasis; WBC: white blood cell; PLR: platelet-lymphocyte ratio; LMR: lymphocyte-monocyte ratio; PNI: prognostic nutritional index; NLR: neutrophil-lymphocyte ratio.

\section{Comparison of LA-TNM and TNM staging systems}

To improve the prognostic value of the TNM staging system, we integrated age and LDH into this system. Age $>57$ and LDH $>245 \mathrm{U} / \mathrm{L}$ were assigned 1 point while age $\leq 57$ and $\mathrm{LDH} \leq 245 \mathrm{U} / \mathrm{L}$ were assigned 0 points. Figure $4 \mathrm{~A}$ and $\mathrm{B}$ show the survival curves stratified by the TNM and LA-TNM staging system, respectively. The log-rank test showed that the $P$ values of these two staging systems were both less than 0.001 . A staging system had a better performance when it had a higher $\chi^{2}$ value and a lower Akaike information criterion(AIC) value in the likelihood ratio test (LRT). The LRT indicated that the $\chi^{2}$ value of the LA-TNM system was significantly higher than that of the TNM system (38.49 vs. $29.29, P<0.001)$. Moreover, the AIC value of the LA-TNM system was significantly lower than that of the TNM system (1437 vs. 1445.32, $P<0.001$ ) (Table 5). Therefore, the LA-TNM system was superior to the TNM system in predicting the overall survival of this cohort of patients in our study.

Table 3. Univariate and multivariate analyses of the prognostic factors for the overall survival of 244 patients with primary duodenum adenocarcinoma.

\begin{tabular}{|c|c|c|c|c|c|c|c|}
\hline \multirow[t]{2}{*}{ Variable } & \multicolumn{2}{|c|}{ Univariate } & \multicolumn{5}{|c|}{ Multivariate } \\
\hline & $\begin{array}{l}\text { Log-rank } \\
\chi^{2}\end{array}$ & P value & B & SE & HR & $95 \% \mathrm{CI}$ & P value \\
\hline $\begin{array}{l}\text { Gender } \\
\text { (male/female) }\end{array}$ & 2.33 & 0.13 & & & & & \\
\hline $\begin{array}{l}\text { Age, years }(>57 / \leq \\
57)\end{array}$ & 12.09 & $0.0005^{*}$ & 0.415 & 0.167 & 1.514 & 1.093 to 2.096 & 0.013 \\
\hline $\begin{array}{l}\text { T category (AJCC } \\
\text { 7th) } \\
\text { (T1/T2/T3/T4) }\end{array}$ & 4.97 & 0.17 & & & & & \\
\hline $\begin{array}{l}\text { N category (AJCC } \\
\text { 7th) }\end{array}$ & 12.06 & $0.0024^{*}$ & & & & & \\
\hline No & & & NA & NA & 1 & Reference & NA \\
\hline N1 & & & 0.230 & 0.540 & 1.258 & $\begin{array}{l}0.4389 \text { to } \\
3.6083\end{array}$ & 0.670 \\
\hline N2 & & & 0.582 & 0.589 & 1.789 & $\begin{array}{l}0.5671 \text { to } \\
5.6424\end{array}$ & 0.324 \\
\hline $\begin{array}{l}\text { M category } \\
\text { (AJCC 7th) } \\
\text { (M0/M1) }\end{array}$ & 61.61 & $\begin{array}{l}< \\
0.0001^{*}\end{array}$ & 0.206 & 0.305 & 1.228 & $\begin{array}{l}0.6777 \text { to } \\
2.2256\end{array}$ & 0.500 \\
\hline $\begin{array}{l}\text { Radical surgery } \\
\text { (Yes/No) }\end{array}$ & 82.54 & $\begin{array}{l}< \\
0.0001^{*}\end{array}$ & -1.396 & 0.172 & 0.248 & 0.177 to 0.346 & $<0.0001$ \\
\hline $\begin{array}{l}\text { TNM (AJCC 7th) } \\
\text { (I-II/ III-IV) }\end{array}$ & 14.94 & $0.0001^{*}$ & -0.278 & 0.587 & 0.757 & $\begin{array}{l}0.2411 \text { to } \\
2.3774\end{array}$ & 0.635 \\
\hline $\begin{array}{l}\text { Degree of } \\
\text { differentiation }\end{array}$ & 7.40 & $0.02^{*}$ & & & & & \\
\hline $\begin{array}{l}\text { Well } \\
\text { differentiation }\end{array}$ & & & NA & NA & 1 & Reference & NA \\
\hline $\begin{array}{l}\text { Moderate } \\
\text { differentiation }\end{array}$ & & & -0.168 & 0.263 & 0.846 & $\begin{array}{l}0.5067 \text { to } \\
1.4114\end{array}$ & 0.523 \\
\hline $\begin{array}{l}\text { Poor } \\
\text { differentiation }\end{array}$ & & & 0.142 & 0.284 & 1.153 & $\begin{array}{l}0.6625 \text { to } \\
2.0070\end{array}$ & 0.616 \\
\hline $\begin{array}{l}\text { NLR }(>2.56 / \leq \\
2.56)\end{array}$ & 3.32 & 0.07 & & & & & \\
\hline $\begin{array}{l}\text { PLR (> 267.5/ } \\
267.5)\end{array}$ & 0.89 & 0.34 & & & & & \\
\hline PNI $(>45.7 / \leq 45.7)$ & 4.98 & $0.03^{*}$ & 0.023 & 0.179 & 1.023 & $\begin{array}{l}0.7214 \text { to } \\
1.4505\end{array}$ & 0.899 \\
\hline $\operatorname{LMR}(>4.7 / \leq 4.7)$ & 2.51 & 0.11 & & & & & \\
\hline LDH $(>245 / \leq 245)$ & 5.25 & $0.02^{*}$ & 0.452 & 0.216 & 1.571 & 1.032 to 2.393 & 0.036 \\
\hline
\end{tabular}

*All variables have a $P$ value less than 0.05 . We entered all the variables with $P<$ 0.05 into multivariate analyses. NA: not applicable; TNM:

Tumour-node-metastasis; AJCC: American Joint Committee on Cancer; WBC: white blood cell; PLR: platelet-lymphocyte ratio; LMR: lymphocyte-monocyte ratio; PNI: prognostic nutritional index; LDH: lactate dehydrogenase; NLR: neutrophil-lymphocyte ratio. 


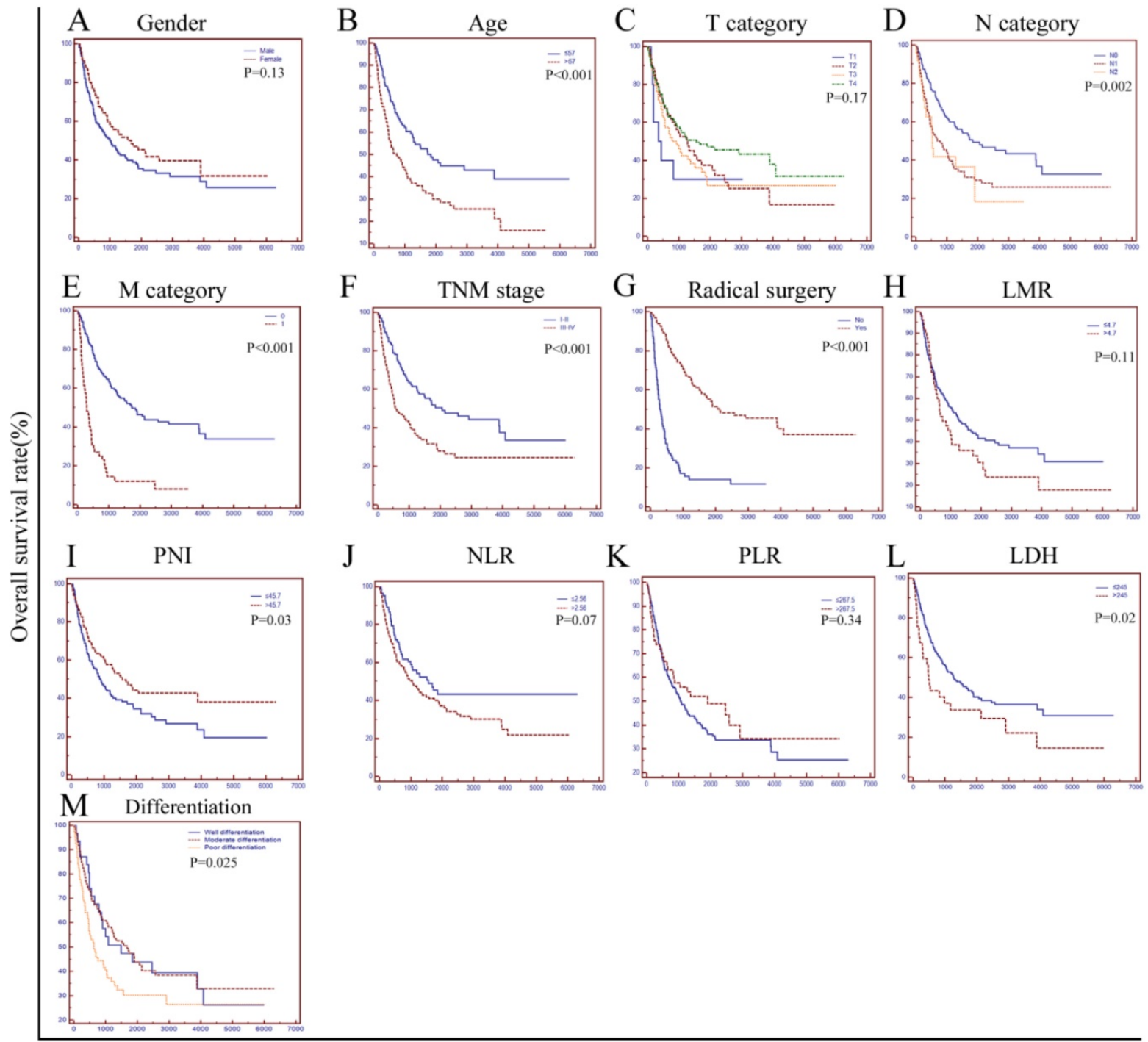

Time after diagnosis(days)

Figure 2. Kaplan-Meier curves stratified by different variables in 244 patients with duodenum adenocarcinoma. OS curves stratified by (A), gender; (B), age; (C), $T$ category; (D), N category; (E), M category; (F), Simplified TNM stage ( I - II vs. III-IV); (G), radical surgery; (H), LMR; (I), PNI; (J), NLR; (K), PLR; (L), LDH, and (M), Degree of pathological differentiation. TNM: tumour-node-metastasis; LMR: lymphocyte-monocyte ratio; PNI: the prognostic nutritional index; NLR: the neutrophil-lymphocyte ratio; PLR: the platelet-lymphocyte ratio; LDH: lactate dehydrogenase.
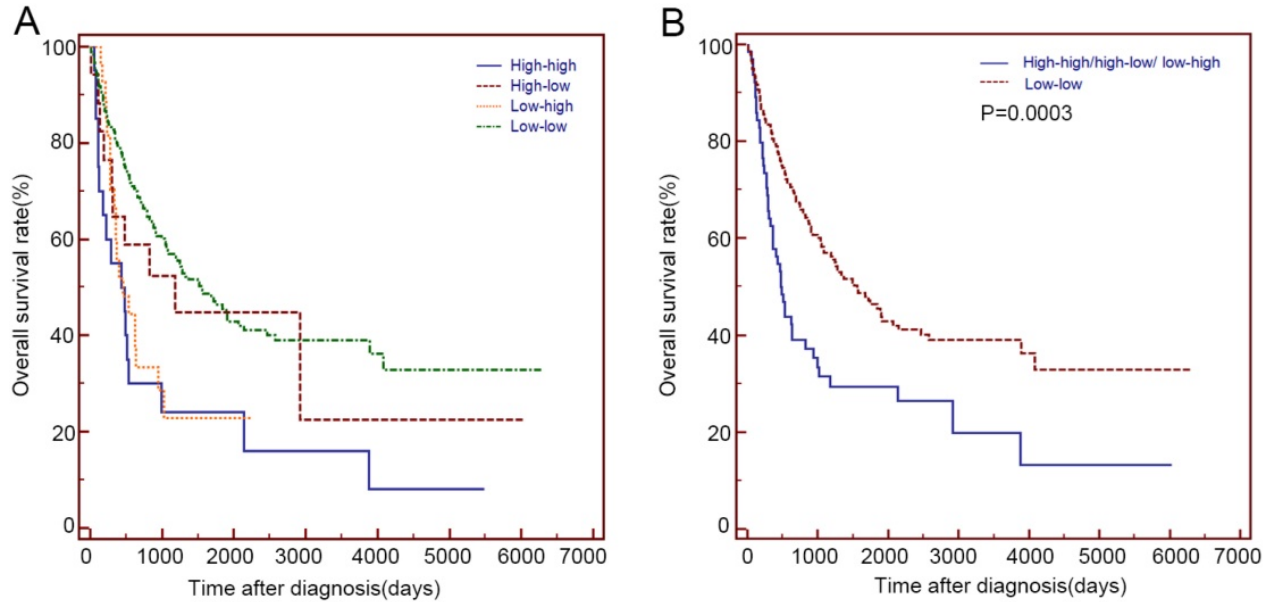

Figure 3. Kaplan-Meier curves for the OS of 244 patients with primary duodenum adenocarcinoma, which were stratified by the values of serum LDH before and after 2 months of radical or palliative treatments. (A) low-low group, low-high group, high-low group and high-high group (B) low-low group and High-high/high-low/ low-high group. 

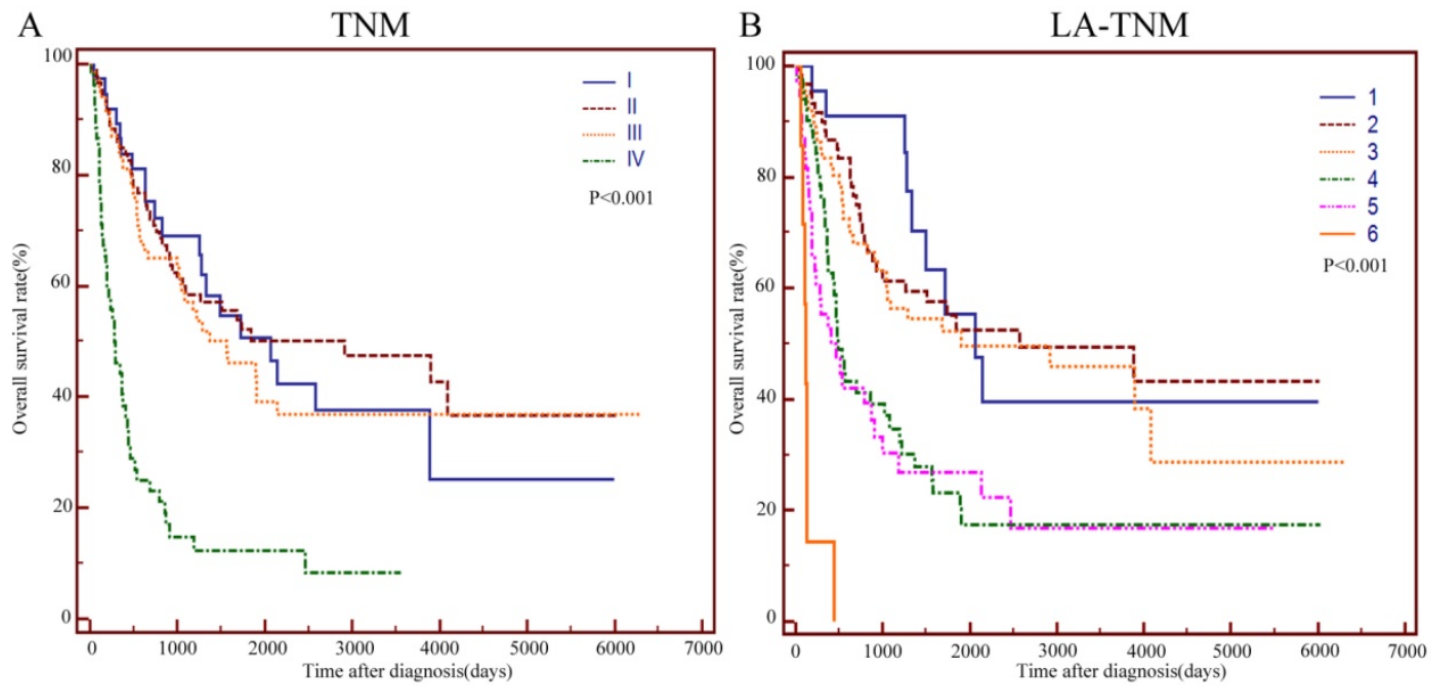

Figure 4. Kaplan-Meier curves for the OS of 244 patients with primary duodenum adenocarcinoma, which were stratified by TNM (A), and LA-TNM (B). TNM: Tumour-node-metastasis; LA: LDH-Age.

Table 4. Overall survival of the different groups based on serum $\mathrm{LDH}$ before and after treatment among the 244 primary duodenum adenocarcinoma patients in the present study.

\begin{tabular}{llll}
\hline Serum LDH & \multicolumn{2}{l}{$\begin{array}{l}\text { Median overall } \\
\text { survival(months) }\end{array}$} & $\begin{array}{l}\text { 95\% CI } \\
\text { (months) }\end{array}$ \\
\hline High-high(n=20) & 14.5 & $1.1-27.9$ & \\
High-low(n=17) & 39.3 & $0-80.6$ & $\begin{array}{l}\text { Vs. high-high: } 0.116 \\
\end{array}$ \\
& 15.4 & $6-24.8$ & Vs. high-high: 0.499 \\
$\begin{array}{l}\text { Low-high(n=27) } \\
\text { Low-low(n=180) }\end{array}$ & 52.1 & $36.7-67.6$ & Vs. high-high: $<0.0001$ \\
$\begin{array}{l}\text { High-high/high-low/ } \\
\text { low-high(n=64) }\end{array}$ & & $11.7-20.1$ & Vs. low-low: $<0.0001$ \\
\hline
\end{tabular}

LDH: lactate dehydrogenase

Table 5. Comparisons of the value of the TNM and LA-TNM systems in the predicting prognosis of the overall survival among the 244 primary duodenum adenocarcinoma patients in the present study.

\begin{tabular}{llll}
\hline Stage system & Likelihood ratio test $\chi^{2}$ & AIC & $p$ value \\
\hline TNM & 29.29 & 1445.32 & $<0.01$ \\
LA-TNM & 38.46 & 1437 & $<0.01$ \\
\hline
\end{tabular}

LA: LDH-Age; TNM: Tumour-node-metastasis; AIC: Akaike information criterion.

\section{Discussion}

Our team previously confirmed that the serum $\mathrm{LDH}$ level was an independent prognostic factor in locally advanced nasopharyngeal carcinoma and oesophageal squamous cell carcinoma $[18,19]$. Other researchers have also confirmed LDH as an independent prognostic factor in pancreatic cancer and colorectal cancer [21, 23]. However, the prognostic significance of LDH in primary duodenum adenocarcinoma has never been assessed hitherto. To the best of our knowledge, this is the first study exploring the prognostic value of $\mathrm{LDH}$ in a large cohort of patients with primary duodenum adenocarcinoma, and we found that elevated baseline serum LDH indicated a poor prognosis.

The tumour microenvironment is frequently hypoxic due to the rapid growth of the tumour cells [13]. In hypoxic conditions, LDH can catalyse pyruvate into lactic acid and plays a key role in anaerobic glycolysis. HIF-1 $\alpha$ and Myc are reported to transcriptionally activate key genes in tumour cellular energy metabolism, such as $L D H$, thereby raising anaerobic glycolysis and producing high levels of lactate $[14,28]$. High levels of lactate will in turn enhance the expression of HIF-1a [14-15]. By modulating metabolism and affecting the tumour microenvironment, the elevation of LDH is closely related to the tumour aggressiveness, metastasis, invasion and tumour burden [14-15]. Consistent with this, our study suggested that a higher LDH level was significantly associated with more distant metastasis, node metastasis, poor differentiation and TNM stage III - IV . On the other hand, a significantly higher median LDH level was observed in patients with a node metastasis, poor differentiation and a TNM stage of III - IV . Researchers revealed that the expression of lactate dehydrogenase- 5 (LDH-5) was associated with the hypoxia-inducible factor (HIF-1a) pathway, angiogenic factor production and poor prognosis in gastric cancer, but not in primary duodenum adenocarcinoma [15]. Further work needs to be done to clarify if these aspects have some distinctive features in primary duodenum adenocarcinoma.

In addition to hypoxia, inflammation is another essential characteristic of tumours, leading to shorter survival in cancer patients [25-27]. Systemic 
inflammation is correlated with hypoxia. Our study demonstrated that the LDH level $(>245 \mathrm{U} / \mathrm{L})$ was significantly associated with a higher NLR.

Previous studies revealed that LDH, HIF-1a and Myc may be regulated by PI3K/Akt/TORC1/HIF pathway [28-30]. In renal cell cancer carcinoma patients with high LDH levels, temsirolimus could provide survival benefits by inhibiting TORC1[28]. Further studies need to be done to clarify whether temsirolimus will benefit primary duodenum adenocarcinoma patients with a higher level of LDH. It is worth mentioning that in colon cancer cells, researchers have found that LDH inhibitors could reduce production of lactate and reduce inflammation-induced changes [31].

An association between a high LDH level and tumour angiogenesis was determined by preclinical studies. HIF-1a was confirmed to increase the serum $\mathrm{LDH}$ level and tumour angiogenesis in vivo. Low levels of LDH may be predictive of the effectiveness of antiangiogenetic therapies [32-33]. In a recent study, $\mathrm{LDH}$ was found to be capable of predicting the benefits from bevacizumab beyond progression in metastatic colorectal cancer. Patients with low serum LDH levels achieved significant PFS benefits from the bevacizumab maintenance treatment [32]. In another study, the colorectal cancer patients with high serum LDH levels did not benefit from regorafenib [33]. Moreover, a retrospective study included 21 unresectable or recurrent duodenum cancer patients to explore the value of the combination of chemotherapy with bevacizumab. The median OS of patients who received a bevacizumab-chemotherapy combination therapy was 21.9 months [34]. The predictive role of $\mathrm{LDH}$ in primary duodenum adenocarcinoma patients receiving antiangiogenetic therapy needs to be further validated.

The cut-off values of LDH varied in the different studies, ranging from 170 to $450 \mathrm{U} / \mathrm{L}$. A recent meta-analysis explored the prognostic value of $\mathrm{LDH}$ in different types of tumours and classified LDH into four groups: <250U/L,250-300U/L,301-400U/L and $>400 \mathrm{U} / \mathrm{L}$. They drew the conclusion that differences among the distinct cut-off points were not statistically $\operatorname{significant}(P=0.99)$ [20]. The ULN of LDH was $245 \mathrm{U} / \mathrm{L}$ in our hospital, and was based on our classification of patients into either the normal LDH $\operatorname{group}(\leq 245 \mathrm{U} / \mathrm{L})$ or the high $\mathrm{LDH} \operatorname{group}(>245 \mathrm{U} / \mathrm{L})$. Through analysis of 244 patients, we identified that patients in the higher LDH group had worse survival outcomes than those in the normal LDH group. Univariate analyses and multivariate analysis confirmed $\mathrm{LDH}$ as an independent prognostic indicator of OS. Higher LDH levels indicated more aggressive and unfavourable outcomes for primary duodenum adenocarcinoma patients. Other independent prognostic factors of OS in the current study were radical surgery and age. It is no doubt that radical surgery is the most important curative treatment for patients with primary duodenum adenocarcinoma. Abeezar et al analysed the prognostic factors of 137 primary duodenum adenocarcinoma patients and found that age and the number of involved lymph nodes were independent prognostic factors [9]. We again confirmed age as a prognostic factor after multivariate analysis. A higher LDH level (>245U/L) indicates apparent hypoxia, exuberant angiogenesis and tumour aggressiveness, which might eventually lead to a worse OS.

To improve the prognostic value of the TNM staging system, we integrated age and LDH into this system to establish the LA-TNM staging system. LRT indicated that the LA-TNM system had a significantly larger $\chi^{2}$ value and a significantly smaller AIC value when compared with the TNM system in predicting the OS. LA-TNM outperformed the TNM system in predicting the overall survival in our selected cohort of patients.

There are several limitations of our study. First, this is a retrospective and single centre study. Although we strictly selected patients in accordance with our inclusion and exclusion criteria, selection bias may still exist. Second, we set the cut-off value of the serum LDH level as the ULN of $245 \mathrm{U} / \mathrm{L}$. As such, external validation cohorts from other hospitals will be needed for further confirmation of higher LDH level(>245U/L) as an independent risk factor for primary duodenum adenocarcinoma. Third, although the theoretical result is economical and convenient to perform, the low proportion of these patients that had a higher level of LDH may lead to limited clinical application. Additionally, a combination of serum biomarkers may be worth exploring. Large scale prospective randomized controlled clinical trials are warranted to further clarify the prognostic value of $\mathrm{LDH}$ in primary duodenum adenocarcinoma. Basic studies should be conducted to study the mechanisms of $\mathrm{LDH}$ and $\mathrm{LDH}$ inhibitors in primary duodenum adenocarcinoma.

In conclusion, our study provided the first demonstration that $\mathrm{LDH}$ is an independent prognostic factor for primary duodenum adenocarcinoma and that elevated baseline serum $\mathrm{LDH}$ levels indicate a poor prognosis.

\section{Abbreviations}

TNM: Tumour-node-metastasis; AJCC: American Joint Committee on Cancer; OS: overall survival; ROC curve: receiver operating characteristics curve; WBC: white blood cell; PLR: 
platelet-lymphocyte ratio; LMR: lymphocytemonocyte ratio; PNI: prognostic nutritional index; LDH: lactate dehydrogenase; NLR: neutrophillymphocyte ratio.

\section{Acknowledgements}

This study was supported by the National High Technology Research and Development Program of China (863 Program), China(2015AA020103); National Natural Science Foundation (31600710, 81372374 and 81602066), Natural Science Foundation of Guangdong (No. 2014A030313205, 2014A030313146 and 2016A03 0313302) and the Project on the Integration of Industry, Education and Research of Guangdong Province (grant no. 2012B091100460), Fundamental Research Funds for the Central Universities (Grant number. 16ykpy25); and The third outstanding young talents training plan and medical scientist program of the Sun Yat-sen Cancer Center.

\section{Competing Interests}

The authors have declared that no competing interest exists.

\section{References}

[1] Chen W, Zheng R, Baade PD, et al. Cancer statistics in China. CA Cancer J Clin. 2015; 2016: 89-171.

[2] V. H. Coupland, H.M. Kocher, D. P. Berry, et al., "Incidence and survival for hepatic, pancreatic and biliary cancers in England between 1998 and 2007," Cancer Epidemiology, vol. 36, no. 4, pp. e207-e214, 2012.

[3] Overman MJ, Hu CY, Kopetz S, Abbruzzese JL, Wolff RA, Chang GJ. A population-based comparison of adenocarcinoma of the large and small intestine: insights into a rare disease. Ann Surg Oncol 2012; 19: 1439-1445

[4] Overman MJ, Varadhachary GR, Kopetz S, et al. Phase II study of capecitabine and oxaliplatin for advanced adenocarcinoma of the small bowel and ampulla of Vater. J Clin Oncol 2009; 27:2598-2603

[5] Solaini L, Jamieson NB, Metcalfe M, et al. Outcome after surgical resection for duodenal adenocarcinoma in the UK. Br J Surg 2015; 102: 676-681

[6] Poultsides GA, Huang LC, Cameron JL, et al. Duodenal adenocarcinoma: clinicopathologic analysis and implications for treatment. Ann Surg Oncol 2012; 19:1928-1935

[7] Zhang S, Cui Y, Zhong B, et al. Clinicopathological characteristics and survival analysis of primary duodenal cancers: a 14-year experience in a tertiary center in South China. Int J Colorectal Dis 2011; 26: 219-226

[8] Wu XY, Chen J, Cao $\mathrm{OH}$, et al. Beclin 1 activation enhances chemosensitivity and predicts a favorable outcome for primary duodenal adenocarcinoma. Tumor Biol. 2013 Apr; 34(2):713-22.

[9] Sarela AI, Brennan MF, Karpeh MS, Klimstra D, Conlon KC. Adenocarcinoma of the duodenum: importance of accurate lymph node staging and similarity in outcome to gastric cancer. Ann Surg Oncol 2004; 11: 380-386

[10] Onkendi EO, Boostrom SY, Sarr MG, et al. 15-year experience with surgical treatment of duodenal carcinoma: a comparison of periampullary and extra-ampullary duodenal carcinomas. J Gastrointest Surg 2012; 16: 682-691

[11] Cecchini S, Correa-Gallego C, Desphande V, et al. Superior prognostic importance of perineural invasion vs. Lymph node involvement after curative resection of duodenal adenocarcinoma. J Gastrointest Surg. 2012 Jan;16(1):113-20; discussion 120 .

[12] Jiang QL, Huang $\mathrm{XH}$, Chen $\mathrm{YT}$, et al. Prognostic Factors and Clinical Characteristics of Patients with Primary Duodenal Adenocarcinoma: A Single-Center Experience from China. BioMed Res Int. 2016; 2016:6491049.

[13] Hsu PP, Sabatini DM. Cancer cell metabolism: Warburg and beyond. Cell. 2008 Sep 5; 134(5):703-7.

[14] Harris AL. Hypoxia -a key regulatory factor in tumor growth. Nat Rev Cancer 2002; 2(1):38-47.

[15] Kolev $Y$, Uetake $H$, and Takagi $Y$, Lactate dehydrogenase-5 (LDH-5) expression in human gastric cancer: association with hypoxia-inducible factor (HIF-1alpha) pathway, angiogenic factors production and poor prognosis. Ann Surg Oncol 2008; 15(8):2336-44

[16] Motzer RJ, Escudier B, Bukowski R, et al. Prognostic factors for survival in 1059 patients treated with sunitinib for metastatic renal cell carcinoma. Br J Cancer. 2013 Jun 25; 108(12):2470-7.
[17] Giroux Leprieur E, Lavole A, Ruppert AM, et al. Factors associated with long-term survival of patients with advanced non-small cell lung cancer. Respirology. 2012 Jan; 17(1):134-42.

[18] Wan XB, Wei L, Li H, et al. High pretreatment serum lactate dehydrogenase level correlates with disease relapse and predicts an inferior outcome in locally advanced nasopharyngeal carcinoma. Eur J Cancer. 2013 Jul;49(10):2356-64.

[19] Wei XL, Zhang DS, He MM, et al. The predictive value of alkaline phosphatase and lactate dehydrogenase for overall survival in patients with esophageal squamous cell carcinoma. Tumor Biol. 2016 Feb; 37(2):1879-87.

[20] Zhang J, Yao YH, Li BG et al. Prognostic value of pretreatment serum lactate dehydrogenase level in patients with solid tumors: a systematic review and meta-analysis. Sci Rep. 2015 Apr 22; 5:9800.

[21] Mekenkamp LJ, Heesterbeek KJ, Koopman M, et al. Mucinous adenocarcinomas: poor prognosis in metastatic colorectal cancer. Eur J Cancer. 2012 Mar; 48(4):501-9.

[22] Weide B, Elsässer M, Büttner P, et al. Serum markers lactate dehydrogenase and $\mathrm{S100B}$ predict independently disease outcome in melanoma patients with distant metastasis. Br J Cancer. 2012 Jul 24; 107(3):422-8.

[23] Yu SL, Xu LT, Qi Q, et al. Serum lactate dehydrogenase predicts prognosis and correlates with systemic inflammatory response in patients with advanced pancreatic cancer after gemcitabine-based chemotherapy. Sci Rep. 2017 Mar 27; 7:45194.

[24] Powles T, Bascoul-Mollevi C, Kramar A, Lorch A, Beyer J. Prognostic impact of LDH levels in patients with relapsed/refractory seminoma. J Cancer Res Clin Oncol. 2013 Aug; 139(8):1311-6.

[25] Li X, Chen ZH, Ma XK, et al. Neutrophil-to-lymphocyte ratio acts as a prognostic factor for patients with advanced hepatocellular carcinoma. Tumor Biol. 2014 Nov;35(11):11057-63.

[26] Li X, Chen ZH, Xing YF, et al. Platelet-to-lymphocyte ratio acts as a prognostic factor for patients with advanced hepatocellular carcinoma. Tumor Biol. 2015 Apr; 36(4):2263-9.

[27] Yingfen Hong, Zhanhong Chen, Li Wei, et al. Identification of the prognostic value of lymphocyte-to-monocyte ratio in patients with HBV-associated advanced hepatocellular carcinoma. Oncology letters 14: 2089-2096, 2017

[28] Armstrong, A. J., George, D. J. \& Halabi, S. Serum lactate dehydrogenase predicts for overall survival benefit in patients with metastatic renal cell carcinoma treated with inhibition of mammalian target of rapamycin. J Clin Oncol. 2012 Sep 20;30(27):3402-7.

[29] Scartozzi M1, Giampieri R, Maccaroni E, et al. Pre-treatment lactate dehydrogenase levels as predictor of efficacy of first-line bevacizumab-based therapy in metastatic colorectal cancer patients. Br J Cancer. 2012 Feb 28; 106(5):799-804.

[30] Vaupel, P. \& Mayer, A. Hypoxia in tumors: pathogenesis-related classification, characterization of hypoxia subtypes, and associated biological and clinical implications. Adv Exp Med Biol. 2014; 812:19-24.

[31] Manerba M, DIIanni L, Govoni M, et al. Lactate dehydrogenase inhibitors can reverse inflammation induced changes in colon cancer cells. Eur J Pharm Sci. 2017 Jan 1; 96:37-44.

[32] Marmorino F, Salvatore L, Barbara C, et al. Serum LDH predicts benefit from bevacizumab beyond progression in metastatic colorectal cancer. Br J Cancer. 2017 Jan; 116(3):318-323.

[33] Kim JW, Dang CV. Multifaceted roles of glycolytic enzymes. Trends Biochem Sci. 2005 Mar; 30(3):142-50.

[34] Takayoshi K, Kusaba H, Uenomachi M, et al. Suggestion of added value by bevacizumab to chemotherapy in patients with unresectable or recurrent small bowel cancer. Cancer Chemother Pharmacol. 2017 Jun 26. 